Brit. J. industr. Med., 1963, 20, 24.

\title{
AETIOLOGY AND PATHOLOGY OF BEAT KNEE
}

\author{
BY \\ W. J. W. SHARRARD \\ From the Royal Infirmary, Sheffield \\ (RECEIVED FOR PUBLICATION JUNE 4, 1962)
}

\begin{abstract}
The object of this study was to determine the mechanism of the production of beat knee and its relation to pathological findings. A clinical study was made among 598 coal-face workers at one large colliery: 579 were examined of whom 233 showed evidence of past or present beat knee. A considerable preponderance of non-inflammatory lesions, most commonly in the prepatellar bursa, was demonstrated. It was concluded that the single common factor in the causation of non-inflammatory or acute recurrent bursitis was the trauma of kneeling.

Experimental investigation of the pressures exerted showed great variations in the pressure on the knee as a whole and on different parts of its weight-bearing surfaces. The view that these localized and extreme variations in pressure may lead to rupture of a vessel found confirmation in the aspiration of blood from the bursae of 29 out of 30 patients. Histological sections from chronically enlarged bursae showed intense fibrosis in the wall of the bursa and sometimes deposition of haemosiderin.

In the management of a new case of acute simple bursitis immediate aspiration of the blood and the instillation of hyalase proved successful in all 12 cases. Aspiration was also successful in certain cases of acute recurrent simple bursitis. Surgical removal of chronically enlarged fibrotic bursae allowed eight out of 12 patients to return to the coal-face.

It is suggested that in order to reduce the incidence of beat knee the use of a knee pad that will not only protect the skin of the front of the knee from trauma by coal particles but will also cushion the knee from the effects of extreme variations in pressure may be desirable.
\end{abstract}

Concern with the incidence of beat knee in kneeling miners has stimulated renewed interest in the condition during the past 10 years (Watkins, 1951 ; Atkins and Marks, 1952; Watkins and Hunt, 1954; Roantree, 1957; Watkins, Hunt, Fernandez, and Edmonds, 1958; Fernandez, 1958). While the definition of beat knee as "a subcutaneous cellulitis or acute bursitis at or about the knee" suffices for the purposes of the Industrial Injuries Act, any study of beat knee must be limited to certain defined clinical and pathological entities. The most recent surveys of beat knee by Roantree (1957) and Watkins et al. (1958) stress a division between inflammatory and non-inflammatory lesions whose aetiology, pathology, and therefore treatment differ.

Using this clinical differentiation as a basis, an investigation was made into beat knee in miners working in the South Yorkshire coal-fields between 1957 and 1959 to try to determine precisely the causes and pathology of the condition and to make recommendations on the management of beat knee in the light of any significant findings.

\section{Incidence of Beat Knee}

The true incidence of beat knee in a population of working miners is extremely difficult to determine. In an attempt to obtain as accurate a picture as possible, 579 out of 598 coal-face workers at Treeton Colliery were examined between September 1957 and November 1958, whether they had symptoms or not. Some of these men and others suffering from various kinds of beat knee were subjected to investigation. Among these 579 men, 233 showed evidence of present or past beat knee at the time of examination. They were classified into three groups: (a) inflammatory lesions, 28; (b) acute simple bursitis, 109; (c) chronic simple bursitis, 96 .

The inflammatory lesions could be subdivided: simple follicular lesions, 8, follicular lesions with sympathetic bursitis, 8 , acute cellulitis, 4 , and septic bursitis, 8 .

Acute simple bursitis cases comprised 54 new acute lesions and 55 acute episodes arising in a chronic bursitis. In this group and in the chronic bursitis group, the prepatellar bursa was affected 
more than twice as often as bursae in the infrapatellar region or compound bursae involving both regions. The distribution of these types of beat knee corresponds closely with that found by Roantree (1957) and Watkins et al. (1958), both as to type and site of lesion.

The last group of men with chronic simple bursitis were not acutely affected and were not, therefore, suffering from beat knee in its strict definition at the time of examination; all this group were at work. Many, but by no means all, gave a history of an earlier attack or attacks of acute bursitis that had subsided but had left them with a persistent swelling of the bursa.

\section{Aetiological Factors}

Almost all previous writers are agreed that the primary aetiological factor in beat knee is kneeling. Beyond this, there are several other factors that might predispose to inflammatory or non-inflammatory beat knee.

Factors Related to Environment.-Watkins et al. (1958) found a close relation between the incidence of beat knee and seam height. The findings in the present survey amply confirmed this opinion and showed that kneeling was as constant a factor in the development of inflammatory beat knee as it was in non-inflammatory lesions. At one pit, where the seams were all over $4 \frac{1}{2} \mathrm{ft}$. in height there were only three cases of beat knee in 1955 and 1956; the average man-shifts worked per month were 42,000 . At another pit where the seams were all between 3 and $4 \mathrm{ft}$. high and the average monthly man-shifts worked were 26,000 , there were, in the same period, 48 cases of beat knee.

The kneeling surface, that is the type of floor and the hardness and size of coal particles, did not appear to play a significant part in the incidence of beat knee. From time to time, however, a local factor such as an awkwardly sloping floor was found to be responsible for a local "epidemic" of beat knee.

The wetness of a pit, its temperature, or its humidity did not appear to have any effect on the incidence of beat knee, though all these factors had a tendency to increase maceration of the skin.

Factors Related to Type of Work.-According to his occupation at the coal-face, a man may have to Jineel mainly in one place (colliers), to crawl interinittently (packers, rippers, belt men), or to crawl constantly (deputies, machine men, drillers, shotiirers). As in previous reports the greatest proportion of men suffering from beat knee were colliers but it was not possible to establish any direct relation between a specific type of work and the incidence of beat knee.

Factors Related to Individual Susceptibility.-Many miners work in the same conditions, yet some willnever develop beat knee. There must, therefore, be some measure of individual susceptibility. Age has been shown by Watkins et al. (1958) to be a factor, in that, in general, the younger the worker the more liable he is to beat knee. The present survey confirms the higher incidence in younger men, for which there may be a number of reasons. The skin and bursae may not yet have become adapted to pressure. The younger miner works faster and throws additional strain on his knees. He often has a young family and does more overtime than many older men. His ligaments take time to stretch so that he cannot sit well back on his haunches and so relieve some of the pressure on his knees. Finally, at the age of 20 greasy skin and superficial septic spots are common.

It seemed possible that some workers were likely, because of the relative prominence of the tibial tubercle or lower pole of the patella, to put more pressure on one or other of these points. To investigate this, 46 patients with beat knee and 46 control cases were radiographed in the kneeling position. No correlation was found to exist between the prominence of the tibial tubercle and the incidence of beat knee in these patients. It was found that the laxity of the anterior cruciate ligaments in a miner's knee allows the femoral condyles to slip forward on the tibia when the knee is flexed, so that the patella is more likely to come into contact with the ground even when the miner is sitting well back on his haunches.

Weight does not appear to affect the incidence of beat knee. Height, on the other hand, may affect the stresses on an individual knee. Thus short men who have to reach further forwards and backwards when shovelling coal wear out all types of protective pad much more quickly than taller men, and it may be that this factor can influence the incidence of beat knee.

Protective Equipment.-One of several varieties of knee pad may be worn by a coal-face worker. Those in common use are of four main types: (a) felt-lined pads, (b) rubber-lined cups, (c) bursa pads, (d) trouser pads. A few workers do not wear any kind of knee pad.

In the cases studied by Watkins et al. (1958), no association was found between the clinical condition, i.e. the type of beat knee encountered, and the type of pad worn. It was apparent from their study that none of the pads prevented or eliminated beat knee 
but there was no evidence to show whether one type of pad might be better than another in the minimization of beat knee.

Each type of pad was found to be liable to defects. The common pad in use in the coal-field investigated was one with an outer protective layer of leather or belting and an inner cushion of felt. In these pads, the inner felt cushion very soon became compressed, hard, and brittle so that they appeared to do little more than protect against pressure from sharp particles or uneven floors. In a number of instances it seemed that the pad itself had been responsible for the production of beat knee by forming a hard ridge on its inner surface.

Rubber-lined cups did not tend to harden, but many miners found that they caused sweating in the region of the knee. With both felt and rubber pads, coal particles were liable to penetrate between the pad and the trouser or knee surface, causing discomfort and skin abrasion.

The bursa pad was designed to try to prevent sweating and collection of coal particles. It consists of a strong outer covering of belting and an inner layer of rubber. The inner layer takes the form of a series of spikes of rubber projecting against the inner aspect of the outer casing and backed by a layer of foam rubber that rests against the knee. The foam rubber layer is perforated by a number of holes. Coal particles that penetrate between the knee and the pad are able to pass through the holes to be discharged between the inner and outer layers. The rubber spikes have a satisfactory cushioning effect when the knee is subjected to pressure. While it appeared to be superior to either of the other types of pad it had the disadvantage of being more expensive and, once it had become worn, it ceased to give adequate protection at precisely the spot where the maximum trauma was being exerted on the knee, and consequently efficiency was reduced considerably.

To assess the usefulness of the bursa pad in men who had suffered recurrent beat knee 24 men were selected, in whom two or more acute recurrences of beat knee had developed during the previous two years. None of them had worn the bursa type of pad before. The immediate results were satisfactory; 22 men reported greater comfort, freedom from coal particles, absence of sweating, and cessation of further trouble with their knees during the next six months, and none during this period developed any recurrence of beat knee. During the subsequent six months, two men developed a recurrence. At the end of a year, further observation was discontinued as most of the pads were beginning to wear significantly.

This experiment suggested that the bursa type of pad might influence the incidence of acute or recurrent beat knee but, since no control cases had been used and there were opportunities for bias in so small a series of selected cases, it was only possible to draw tentative conclusions.

\section{Experimental Investigation of Pressure on Knee in Kneeling Miners}

Since the single common factor that can be shown to influence the incidence of any type of beat knee is kneeling, an investigation was made to try to discover the forces and pressures exerted upon the knee in a miner in the course of his work.

The pressures were investigated in two ways. First, variations in pressure as a whole on the knees and toes of miners who were shovelling were recorded by placing the kneeling subject on weighing scales positioned under each knee and under the toes (Fig. 1). Secondly, the distribution of local pressures on the knee itself was investigated by means of an apparatus consisting of four plastic tubes filled with oil and attached to four pressure gauges (Fig. 2). The tubes were so arranged that they could be applied under the knee of a kneeling man so as to record pressure (a) under the tibial tubercle, (b) in the interval between the tibial tubercle and the patella, (c) under the distal pole of the patella, and (d) under the body of the patella. Readings were taken of the pressures on these points in men engaged in coal-face work.

The results in a miner engaged in shovelling are shown in Fig. 3. The procedure of shovelling was divided into seven phases:

1: From the resting position, sitting on the heels, to apply the shovel to the coal.

2: Applying the face of the shovel into or under the coal.

3: Drawing back coal on the shovel.

4: Lifting the loaded shovel.

5: Transference of the coal load to conveyor belt.

6: Discharge of coal from the shovel.

7: Return to the resting position.

A right-handed collier weighing $189 \mathrm{lb}$. $(85.7 \mathrm{~kg}$.) was throwing coal from right to left for a distance of $9 \mathrm{ft}$. At rest, the distribution of his weight was $49 \mathrm{lb}$. $(22 \cdot 2 \mathrm{~kg}$.) through the left tibial tubercle, $56 \mathrm{lb}$. $(25.4 \mathrm{~kg}$.) through the right tibial tubercle, and $84 \mathrm{lb}$. $(38 \cdot 1 \mathrm{~kg}$.) through the toes (Fig. 1). The graph shows the extreme variations in pressure between the two knees during the various phases of shovelling. In particular, during the transfer of thi: coal load onto the belts, the left knee was taking almost the whole of the body weight plus the weight of the shovel and the coal, while the pressure on the 


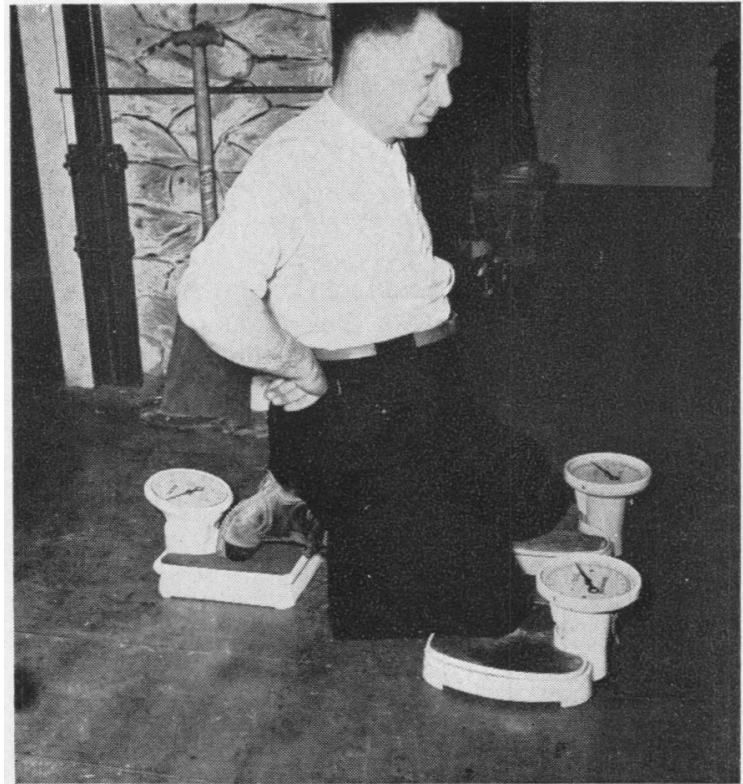

Fig. 1.-Weighing scale for measuring pressures in kneeling miners.

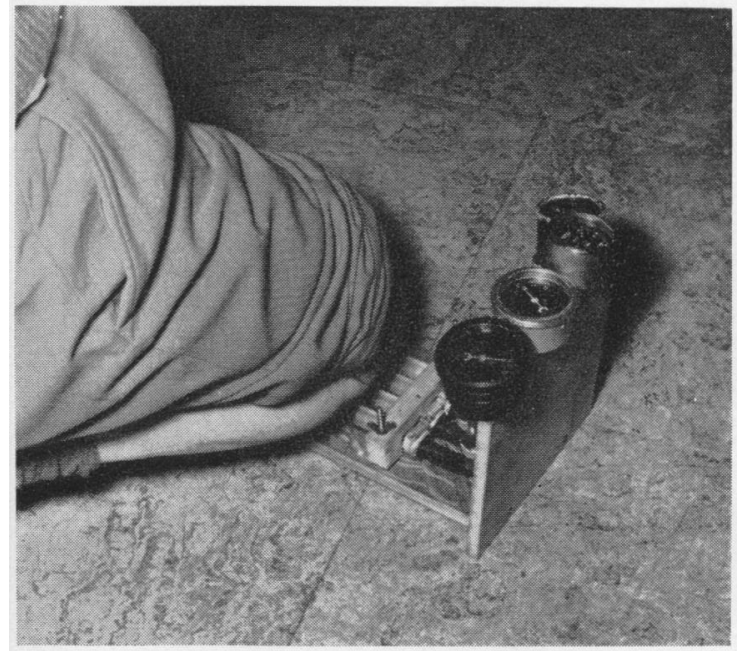

Fig. 2.-Apparatus for measuring local pressure on the knee.

Fig. 3.-Graph to show pressure exerted on each knee and on the toes during a cycle of shovelling.

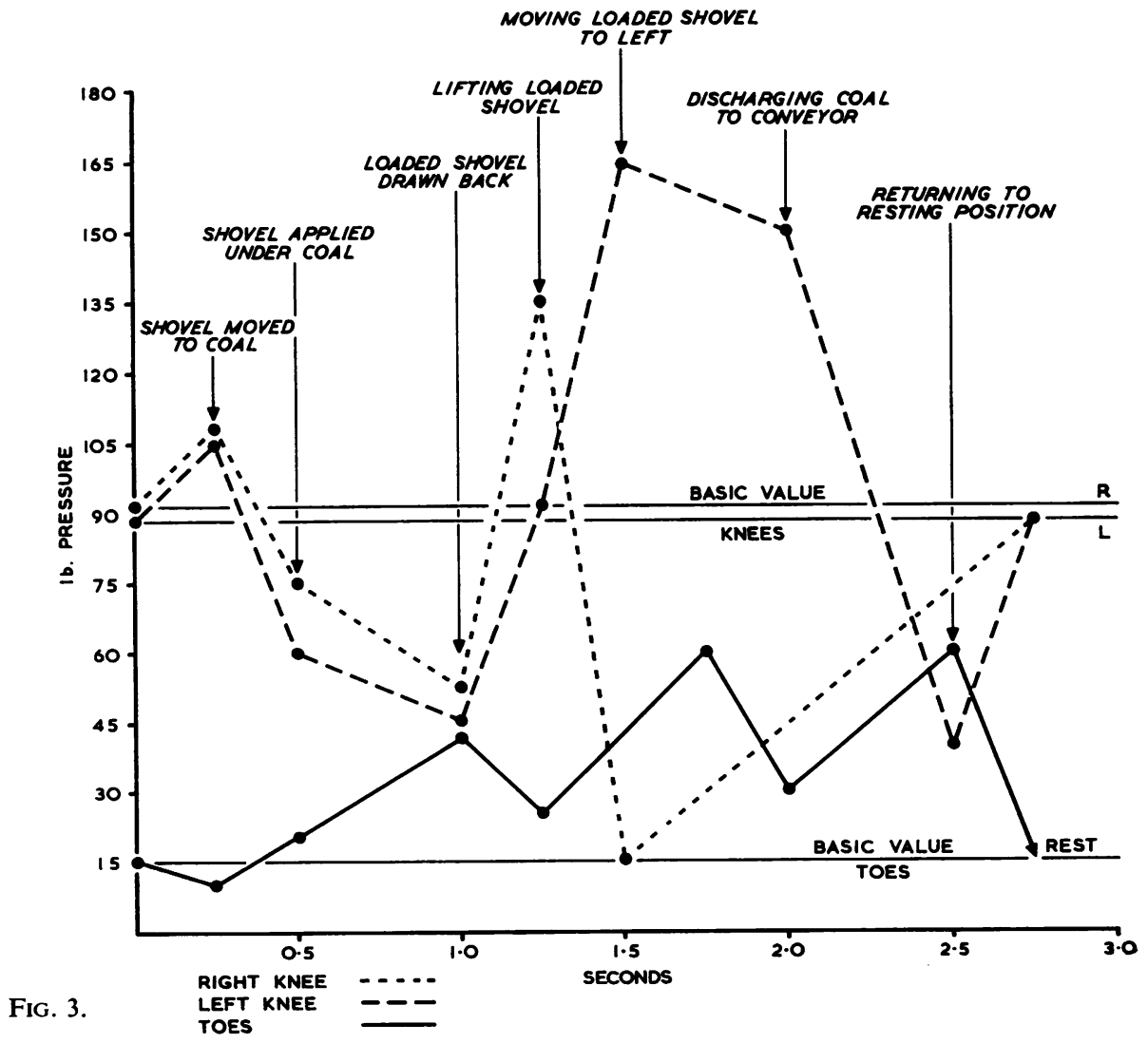

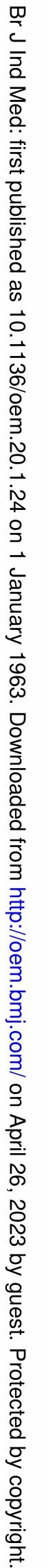


right knee diminished rapidly to a very low figure. The cycle of events took place at every shovelling, i.e. every $2 \cdot 5$ seconds.

Experiments with the oil-pressure gauges showed that different portions of the knee were subjected to pressures at different times. When the subject was at rest sitting on his heels, the pressure was being taken almost entirely on the tibial tubercle. As he moved forward to shovel, the patellar readings increased in value. The tibial tubercle to patella interval showed a slight increase and then fell to zero. As the subject returned his weight to the heels, there was a sudden marked increase of pressure as weight passed across the interval between the patella and the tibial tubercle again.

The combined results of these experiments show very clearly that considerable variations in pressure on the knee as a whole and on different parts of the weight-bearing surfaces of the knee are taking place all the time as men are working. Pressure can rise suddenly over a localized area from zero to as much as $200 \mathrm{lb}$. $(90.70 \mathrm{~kg}$. $) /$ in. ${ }^{2}\left(2.54 \mathrm{~cm} .{ }^{2}\right)$ in a very brief interval of time. The sites of maximum wear of knee pads add evidence to this analysis of the distribution of pressure on the knee.

\section{Pathology of Beat Knee}

Inflammatory Lesions.-In the majority of cases, a study of the pathology of inflammatory lesions shows nothing more unusual than an infection of a hair follicle, sometimes with local abscess formation, or a diffuse cellulitis of the type encountered in streptococcal infections. In 26 cases, bacteriological investigation was done on the infecting organism. All but two grew Staphylococcus pyogenes on culture; fluid aspirated from the bursa through an uninfected area was sterile. The two exceptions had extensive cellulitis and oedema of the leg; the fluid aspirated from the bursa was purulent and grew a Streptococcus haemolyticus, Lancefield Group A. One of these two cases also had multiple septic spots, but the organism grown from these spots was found to be a coagulase-negative staphylococcus.

The finding that a coal particle is not infrequently discharged from a localized abscess lends weight to the view that some of these lesions arise as a result of the penetration of small coal particles into the skin. Any effusion that develops coincidentally in a nearby bursa usually subsides rapidly with the disappearance of the adjacent infective lesion, and it seems likely that its nature corresponds to the sympathetic effusion that is not infrequently seen in the knee joint in cases of osteomyelitis of the lower end of the femur in which the knee joint itself has remained uninfected. In one case of true septic bursitis, which is rare, the bursa contained both blood and pus, and there was a strong inference that blood effusing into the bursa had become infected from a nearby follicular lesion.

Acute Simple Bursitis.-A torn semilunar cartilage was removed from kneeling miners and biopsy specimens were taken from normal prepatellar bursae in non-miners and from prepatellar bursae in kneeling miners who showed no evidence of previous beat knee. A notable feature of a normal bursa was that its wall abounded in large and delicate blood vessels.

Biopsy specimens from the infrapatellar region almost always failed to show any bursal tissue in non-miners. To discover whether the infrapatellar bursa was a normal structure, an exploration was made at necropsy of the region between the skin and the tibial tubercle and patella tendon in 36 subjects selected at random, none of them having been miners. In no case was it possible to discover any bursal tissue. The conclusion is that while the prepatellar bursa is a normal structure, the infrapatellar bursa is an adventitious bursa that develops in those who subject this area of their knee to pressure by kneeling. Support for this view is given by the fact that normal subcutaneous bursae such as the prepatellar and olecranon bursae occur in sites where the skin moves over the surface of the bone on movement of the joint. This is true of the prepatellar region but it is not true of the infrapatellar region; the skin does not move over the tibial tubercle on flexion movement of the knee joint.

Aspiration of a bursa affected by acute simple bursitis in a patient who had never had a previous acute incident almost always produced sterile fluid containing a greater or lesser proportion of blood. Among 30 patients who developed acute bursitis in a previously unaffected bursa, only five of whom had a history of direct trauma, pure blood was obtained on aspiration in 29. A mixture of blood and pus was aspirated in one patient. In patients suffering from an acute episode superimposed on a chronic swelling of a bursa, blood-stained bursal fluid was frequently obtained. It seemed that the more long-standing the swelling of the bursa, the less likelihood was there that blood would be found at any acute recurrence.

Opportunities to obtain histological examination of an acute simple bursitis within a short time of the onset in a previously unaffected bursa are rare. In one case, an acute swelling of the prepatellar bursa developed after kneeling, and aspiration showed it to contain pure blood. At the patient's request, an operation was performed 24 hours later to remove 


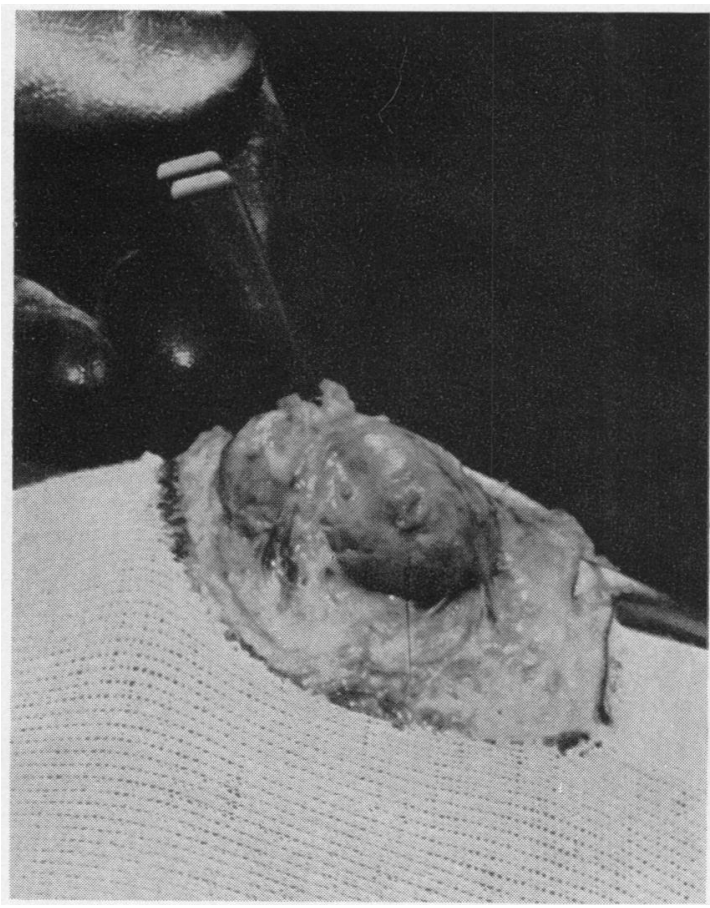

Fig. 4.-Removal of a prepatellar bursa 24 hours after onset of acute simple bursitis. The bursa contained pure blood under tension.

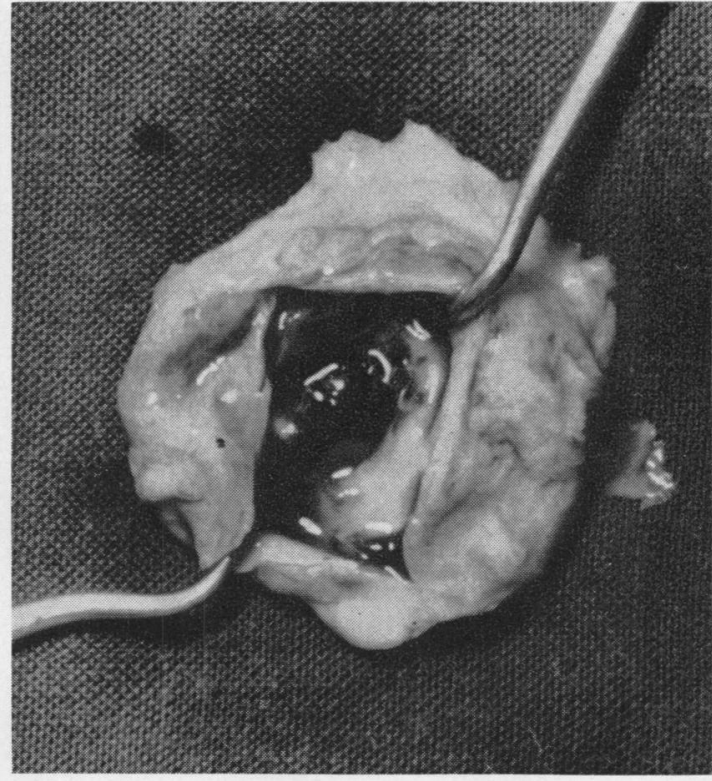

Fig. 5.-Excised bursa opened to show clotted blood adhering to the wall.

FIG. 6.-Histological section $(\times 75)$ of bursal wall in acute simple bursitis showing rupture of a large thin-walled vessel.

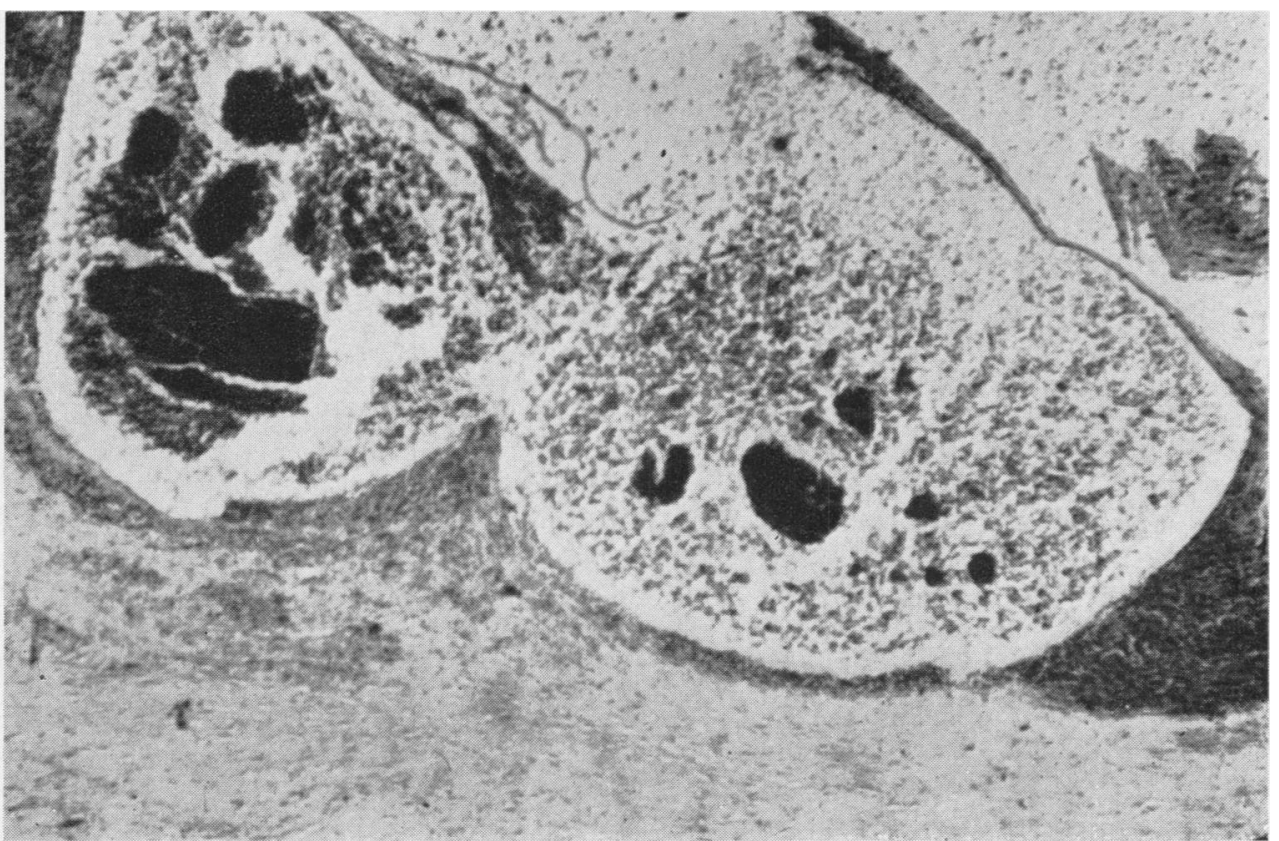

FIG. 6. 
the bursa (Figs. 4 and 5). Histological sections showed that a large vessel in the wall of the bursa had ruptured (Fig. 6) and confirmed the presence of other large vessels lying immediately below the bursal lining. These findings show that the mechanism for the development of many acute cases of simple bursitis is one of rupture of one of the large vessels in the lining wall of the bursal sac.

Chronic Simple Bursitis.-Histological sections taken from chronically enlarged bursae show an intense fibrosis in the wall of the bursa, obliteration of many of the large vessels, and a simple synovial lining. Some bursae from long-standing cases may show considerable haemosiderin deposition in the wall. Evidence from the behaviour of haemarthroses supports the concept of an initial haemorrhage followed by an aseptic inflammatory reaction and slow resolution.

It is probable that the normal absorption mechanism for bursal fluid is completely disorganized. Particles of small molecular weight diffuse through synovial membrane without difficulty, but those of larger molecular weight, including blood proteins, do so more slowly. The breakdown products of blood are especially slow in diffusing, and haemosiderin is often trapped in the membrane for long periods after an injury. The larger particles are removed by phagocytosis, the phagocytes being the lining endothelial cells, and the lymphatic channels easily become blocked. The absorption of fluid is therefore considerably slowed and, if the lesion is extensive, permanent damage occurs. Such a mechanism would account for the much slower rate at which aseptic bursitis subsides compared with septic bursitis and would account for some of the liability to recur and to persist after an initial haemorrhage or after several haemorrhages.

\section{Management of Beat Knee}

The ideal condition would be one in which beat knee was completely prevented, but it seems likely that, whatever is done, some miners will develop beat knee because of the need to kneel. Simple measures of cleanliness and hygiene, especially in young men with greasy skin and liability to superficial staphylococcal infection, can (Atkins and Marks, 1952) reduce the incidence of inflammatory lesions. The use of a pad that minimizes the collection of coal particles and maceration of the skin by sweating, as does a bursa type of pad, is also likely to diminish the incidence of inflammatory cases of beat knee. The same type of pad, with its more effective cushioning of the extreme variations in pressure on the knee, demonstrated in the pressure experiments, might be expected to reduce the liability to acute simple bursitis which is a haemobursa.

In the management of recent acute simple bursitis, immediate aspiration of the blood before it has time to organize and to create the conditions likely to lead to a chronic bursal effusion seems likely to give the best results. In 12 cases the blood was aspirated within the first three days, and hyalase in $1 \mathrm{ml}$. fluid was instilled. In these patients only two needed further aspiration, and none proceeded to a chronic state.

Aspiration may also be successful in some cases of acute recurrence in a chronic simple bursitis, but the long-term results are not so reliable. Aspiration in a severe case of chronic simple bursitis is seldom of value, and if the provision of adequate pads is not sufficient to allow a man to return to work surgical excision of the bursa is the only remaining alternative. If the operation is to be satisfactory it is essential that the incision be a generous one skirting the lateral margin of the bursa and avoiding any of the weight-bearing area. Care must be taken to preserve cutaneous innervation, as far as possible. The skin flap, especially in the region of the infrapatellar bursa, may be very thin, but healing has been uneventful in most cases. With these precautions, eight out of 12 patients have returned to coal-face work, a higher proportion than that recorded in a similar investigation by Roantree (1957).

\section{Discussion}

The results of clinical, experimental, and histological investigation explain several features of beat knee that have previously been open to question. The immediate cause of an acute simple bursitis appears to be rupture of one of the large and delicate vessels in its wall. The results of pressure experiments in working miners suggest that the intense and repeated variations in pressure on different parts of the knees are more than sufficient to account for the mechanism of this rupture. The greater incidence of prepatellar over infrapatellar bursitis is partly explained by the fact that the infrapatellar bursa is not a normal structure and must develop in response to pressures of kneeling before subsequent haemorrhagic events convert it into a haemobursa. The pressure experiments reveal how, in the course of normal work, pressure is exerted on the prepatellar bursa during shovelling and other working activities.

All these findings suggest that any attempt to minimize the incidence of beat knee must be directed to cushioning as far as possible not only the effects of direct trauma of coal particles and hard surfaces on the front of the knee but also the varia- 
tions in pressure upon these surfaces. Fernandez (1958) found that men suffering from acute beat knee could often continue to work provided that a large mine dressing was placed in front of the knee between it and their normal knee pad. The disadvantage of a bulky protection of this kind is instability, but it does seem to indicate that appropriate distribution of forces in the front of the knee can minimize trauma. The greater protection afforded by the bursa type of pad seems to indicate its use by those suffering from acute beat knee and possibly also in the minimization of trauma to and haemorrhage into the bursae.

The effect of immediate aspiration of an acute haemobursa has its parallel in haemarthrosis in which early aspiration can minimize or prevent the deleterious effects of the later action of the products of breakdown upon the lining tissues of the joint.
I wish to acknowledge the assistance given by $\mathrm{Mr}$. D. M. Caird, and Mr. J. J. McKessack for much of the field work and for carrying out some of the experiments. My thanks are also due to Dr. J. M. Rogan, Dr. J. S. McLintock, and Mr. F. D. K. Liddell of the Medical Service of the National Coal Board for much helpful criticism.

The co-operation of the management of Bursa Pads Ltd., Nottingham, was also much appreciated. The work was undertaken with the assistance of a grant from the National Coal Board.

\section{REFERENCES}

\begin{tabular}{l} 
Atkins, J. B., and Marks, J. (1952). Brit. J. industr. Med., 9, 296. \\
Fernandez, R. H. P. (1958). ibid., 15, 110. \\
Roantree, W. B. (1957). ibid., 14, 253. \\
Watkins, J. T. (1951). Practitioner, 167, 257. \\
\hline and Hunt, T. A. (1954). Med. Press, 232, 5. \\
\hline J. industr. Med., 15, 105.
\end{tabular}

The October (1962) issue contains the following papers:-

Protective Clothing as a Factor in the Dust Hazard of Potters. W. A. Bloor and A. Dinsdale

A Dust Dispersal Machine for Inhalation Experiments. A. M. Fisher and Helen Christie

Hard Metal Disease. A. O. Bech, M. D. Kipling, and J. C. Heather

Pulmonary Fibrosis and Encephalopathy Associated with the Inhalation of Aluminium Dust. A. I. G. Mclaughlin, G. Kazantzis, E. King, Donald Teare, R. J. Porter, and R. Owen

The Energy Expenditure of Coal-miners at Work. P. W. Humphreys and A. R. Lind with K. S. SweEtLAND Toxic Properties of Dialkylnitrosamines and Some Related Compounds. D. F. Heath and P. N. MAGeE

A Study of Acrylonitrile Poisoning in Relation to Methaemoglobin-CN Complex Formation. LADISLAUS MaGos

Effects of Hyoscine and Meclozine on Vigilance and Short-term Memory. W. P. Colquhoun

The Beginnings of Industrial Medicine in England. H. BuEss

Book Reviews

Index to Volume 19

A number of copies are still available and may be obtained from the Publishing Manager, British Medical Association, Tavistock Square, W.C.1. price 17s. $6 d$. 